OPEN ACCESS

Edited by:

Andrew Harkin,

Trinity College Dublin, Ireland

Reviewed by:

Annette Horstmann,

Max Planck Institute for Human

Cognitive and Brain Sciences,

Germany

Anton Lord,

QIMR Berghofer Medical Research Institute, Australia

*Correspondence: Nathalie Just

nathalie.just@ukmuenster.de

Specialty section: This article was submitted to

Systems Biology,

a section of the journal

Frontiers in Physiology

Received: 12 October 2016 Accepted: 11 January 2017

Published: 31 January 2017

Citation:

Just N and Sonnay S (2017) Investigating the Role of Glutamate and GABA in the Modulation of

Transthalamic Activity: A Combined fMRI-fMRS Study. Front. Physiol. 8:30.

doi: 10.3389/fphys.2017.00030

\section{Investigating the Role of Glutamate and GABA in the Modulation of Transthalamic Activity: A Combined fMRI-fMRS Study}

\author{
Nathalie Just ${ }^{1,2 *}$ and Sarah Sonnay ${ }^{3}$ \\ ${ }^{1}$ CIBM-AlT core, Ecole Polytechnique Fédérale de Lausanne, Lausanne, Switzerland, ${ }^{2}$ University Hospital Münster, Münster, \\ Germany, ${ }^{3}$ LIFMET, Ecole Polytechnique Fédérale de Lausanne, Lausanne, Switzerland
}

The Excitatory-Inhibitory balance (EIB) between glutamatergic and GABAergic neurons is known to regulate the function of thalamocortical neurocircuits. The thalamus is known as an important relay for glutamatergic and GABAergic signals ascending/descending to/from the somatosensory cortex in rodents. However, new investigations attribute a larger role to thalamic nuclei as modulators of information processing within the cortex. In this study, functional Magnetic Resonance Spectroscopy (fMRS) was used to measure glutamate (Glu) and GABA associations with BOLD responses during activation of the thalamus to barrel cortex (S1BF) pathway at 9.4T. In line with previous studies in humans, resting GABA and Glu correlated negatively and positively respectively with BOLD responses in S1BF. Moreover, a significant negative correlation $(R=-0.68, p=0.0024)$ between BOLD responses in the thalamus and the barrel cortex was found. Rats with low Glu levels and high resting GABA levels in S1BF demonstrated lower BOLD responses in S1BF and high amplitude BOLD responses in the thalamus themselves linked to the release of high GABA levels during stimulation. In addition, early analysis of resting state functional connectivity suggested EIB controlled thalamocortical neuronal synchrony. We propose that the presented approach may be useful for further characterization of diseases affecting thalamocortical neurotransmission.

Keywords: fMRS, BOLD, resting state, barrel cortex, thalamus, glutamate, GABA

\section{HIGHLIGHTS}

- Thalamic and barrel cortex fMRS, BOLD, and resting state fMRI were conducted.

- [GABA] inversely correlated with BOLD responses in S1BF.

- The stimulated thalamic neurochemical profile differed from that of S1BF.

- Regulation of functional connectivity between thalamus and cortex was suggested.

\section{INTRODUCTION}

There is a growing body of evidence showing that both excitatory and inhibitory neurons govern the hemodynamic response to increased neuronal activity (Kocharyan et al., 2008; Enager et al., 2009; Logothetis et al., 2010; Lecrux et al., 2011) at the same time as they need "fuel" to work. As a consequence of this activity, neurons release glutamate (Glu), and $\gamma$-amminobutyric acid (GABA) neurotransmitters. Glu and GABA as well as other metabolites (Lactate, Aspartate, Glucose..) play a significant role in modulating brain activity during both stimulus-induced activity and "intrinsic" 
ongoing activity (Duncan et al., 2014). More than 10 years ago, Chen et al. (2005) demonstrated that GABA enhancement can decrease Blood Oxygen Level dependent (BOLD) signal amplitudes in the rat forepaw cortex. It is now well-admitted that resting state GABA concentrations correlate negatively with Blood Oxygen Level dependent responses in various regions of the human brain (Northoff et al., 2007; Donahue et al., 2010; Muthukumaraswamy et al., 2012; Bednařík et al., 2015). Morover, the modulation of neuronal activity by resting glutamate concentrations was also shown (Kapogiannis et al., 2013) although studies examining the relationship were sparser.

Another interesting approach for an improved understanding of neuroimaging signals may be to investigate how neuronal activity in a specific region of the brain influences the rest of its interconnected network. In particular, derivation of metabolic relationships between different regions in a neural network may be informative. Duncan et al. (2011) assessed functional connectivity between regions of the human anterior cingulate cortex (ACC) and related BOLD responses to [Glu] in one region but not the other thus demonstrating the influence of deactivation of one region on activation of the other through a glutamatergic pathway. Although the number of studies investigating how excitatory and inhibitory neurotransmitters mediate BOLD responses is growing, the potential of this relatively new approach remains to be explored.

In rodents, the thalamus is recognized as one of the most important brain areas for driving cortical processing (Devor et al., 2005; Poulet et al., 2012; Feldmeyer et al., 2013). Thalamic nuclei such as the ventral posteromedial thalamic nucleus (VPM) were often described as important relays for glutamatergic and GABAergic functional information processing of the ascending (and descending) response to whisker or forepaw stimulations. Notably, in models of absence epilepsy, the thalamus revealed to be more than a passive resonator for spike-wave-discharges maintenance while directional nonlinear couplings between cortex and thalamus were suggested in conjunction with several glutamatergic and GABAergic receptor dysfunction (Lüttjohann and van Luijtelaar, 2015). Other pathologies such as schizophrenia could also originate from thalamic dysregulation and functional disconnectivity again linked to excitatory-inhibitory balance (EIB) dysfunction (Behrendt, 2006). In particular, several optogenetics studies suggested that glutamatergic and GABAergic modulations of neural activity could benefit the investigation of diseases affecting thalamo-cortical neurotransmission. In order to examine this proposition under pathological conditions, normal biochemical modulatory activities between cortex and thalamus must be characterized.

In the present study, the neurochemical profiles within the barrel cortex (S1BF) and the thalamus of rats before

\footnotetext{
Abbreviations: fMRS, Functional Magnetic Resonance Spectroscopy; BOLD, Blood Oxygen Level Dependent; TGN, trigeminal Nerve; S1BF, Primary somatosensory Barrel Field cortex; [Glu], glutamate concentration; [GABA], $\gamma$-amminobutyric acid concentration; [Lac], Lactate; [Gln]: Glutamine. VPM, ventral posteromedial thalamic nucleus POm, Posteromedian Thalamic Nucleus; VOI, Volume of Interest; VASO, vascular space occupancy; CBF, cerebral blood flow; fWE, family wise error.
}

and during stimulation of the trigeminal nerve as well as BOLD responses were measured. A correlation study was then conducted to characterize associations between BOLD, [Lac], [Glu], and [GABA] in both thalamic and cortical structures and between them. We expected to verify that BOLD responses and [GABA] were negatively correlated within cortical and subcortical areas while positive correlations between BOLD and stimulation-induced changes in glutamate levels $(\Delta[\mathrm{Glu}])$ as well as lactate $(\Delta[\mathrm{Lac}])$ were envisaged in cortex. The influence of glutamate and GABA on thalamocortical neurotransmission were discussed.

\section{MATERIALS AND METHODS}

\section{Animals}

All studies were performed following the approval of Service de la consommation et des affaires vétérinaires du canton de Vaud (Switzerland) and according to the federal guidelines of the Animal Care and approved by the local authority (EXPANIM-SCAV). Male Sprague-Dawley rats $(n=15,350$ \pm 40 g; Charles River, L'Arbresle, France) under isoflurane anesthesia (2-3\%) vaporized in $30 \% \mathrm{O}_{2}$ in air were intubated, and mechanically ventilated. Two femoral arteries and one femoral vein were catheterized for blood gas sampling and blood pressure measurements as well as $\alpha$-chloralose (an initial intravenous dose of $80 \mathrm{mg} / \mathrm{kg}$ was administered followed by a continuous intravenous infusion of $27 \mathrm{mg} / \mathrm{kg} / \mathrm{h}$ at a rate of $2 \mathrm{ml} / \mathrm{h}$ ) and pancuronium administrations. Respiration rate was monitored through a pillow (SA Instruments, Stony Brook, NY, USA) placed underneath each rat. Temperature was measured using a rectal sensor and regulated via control of the temperature of water flowing through tubing covering the body of each rat and linked to a temperature-regulated bain-marie. Less than $300 \mu \mathrm{l}$ of arterial blood were sampled every $30 \mathrm{~min}$ and blood parameters directly measured using an AVL blood gas analyzer (Dotmed, USA). Mean Arterial blood pressure (MABP) was measured continuously using a transducer attached to the femoral artery catheter. Body temperature and blood parameters were maintained at physiological levels $\left(\mathrm{T}=37.5^{\circ} \mathrm{C} \pm 0.5^{\circ} \mathrm{C}\right.$; $\mathrm{pH}=7.4 \pm 0.05, \mathrm{pCO} 2=39.7 \pm 7 \mathrm{mmHg}$ and $\mathrm{MABP}=$ $148.9 \pm 11 \mathrm{mmHg}$ ) throughout each experiment. An intravenous femoral injection of Pancuronium Bromide (Sigma, Switzerland) of $0.7 \mathrm{ml}$ per hour was performed to minimize tremors. Rats were positioned in a dedicated stereotactic holder equipped with ear and bite bars which was tilted in the magnet $\left(30-45^{\circ}\right)$ for a better positioning of voxels for fMRS over the barrel cortex. fMRS measurements were conducted sequentially in thalamus and S1BF followed by BOLD fMRI in 15 rats. The resting-state $\mathrm{fMRI}$ analysis is presented as supplementary data in the present study.

\section{Trigeminal Nerve Stimulation (TGN)}

Electrodes were percutaneously inserted in the left infraorbital nerve. Electrical stimulation of the left trigeminal nerve (TGN) was performed using an external stimulator (WPI, Stevenage, UK) as described in Just et al. (2010). The paradigm of stimulation ( 1 minOFF-1 minON...) for both fMRI and fMRS 
was repeated for $32 \mathrm{~min}$ with pulse duration of $0.5 \mathrm{~ms}$, stimulation frequency of $1 \mathrm{~Hz}$ and stimulation current amplitude of $2 \mathrm{~mA}$ (Sonnay et al., 2015).

\section{Magnetic Resonance Experiments}

Experiments were described schematically in Figure 1. Experiments were performed on an actively shielded $9.4 \mathrm{~T} / 31 \mathrm{~cm}$ bore magnet (Agilent, USA) with $12 \mathrm{~cm}$ gradients and a surface coil. Shims were adjusted using FAST(EST)MAP (Gruetter and Tkác, 2000).

\section{Functional MR Spectroscopy}

Localized proton spectroscopy was performed using Spin Echo Full Intensity Acquired Localized Sequence (SPECIAL, TE = $2.8 \mathrm{~ms}$, TR $=4 \mathrm{~s}$ ) (Mlynárik et al., 2006) in 15 rats. The voxel of interest for the thalamus was chosen by reference to the Paxinos and Watson atlas (Paxinos and Watson, 1998) so that it encompasses the VPM and POm structures $(n=12)$. Its size was $3 \times 3 \times 4 \mathrm{~mm}^{3}$ and it was shimmed down to a linewidth of $12 \pm$ $2 \mathrm{~Hz}$. In the barrel cortex, the VOI size was $1.5 \times 3 \times 5 \mathrm{~mm}^{3}$ and was shimmed down to a linewidth of $10 \pm 2 \mathrm{~Hz}(n=15)$. For each VOI, ${ }^{1} \mathrm{H}$ spectra were acquired during 32 -min of rest followed by $32 \mathrm{~min}$ of TGN stimulation corresponding to 480 scans ( 30 $\times 16$ ) per period. The water signal was suppressed using the VAPOR module containing a series of seven $25 \mathrm{~ms}$ asymmetric variable power RF pulses with optimized relaxation delays. To improve the signal localization, three modules of outer volume saturation (OVS) were interleaved with the water suppression pulses. The raw ${ }^{1}$ HMRS spectra corrected for frequency drift and summed were used for LCModel analysis with a basis set of 21 simulated metabolites (Macromolecules: Mac; Scyllo-inositol: Scyllo; Ala: Alanine; Ascorbate: Asc; Aspartate: Asp; $\beta$-hydroxybutyrate: bHb; Glycerophosphocholine: GPC, Phosphocholine: PCho; Creatine: Cr; Phosphocreatine; $\gamma$ aminobutyric acid: GABA; Glucose: Glc; Glutamine: Gln; Glutamate: Glu; Glutathione: GSH; myo-inositol: Ins; Lactate: Lac; N-acetylaspartate: NAA; N-Acetylaspartylglutamic acid:NAAG; Phosphatidylethanolamines: PE; Taurine: Tau). Absolute metabolite concentrations were obtained using unsuppressed water signal (8 averages) as an internal reference. The Cramer-Rao lower bounds were used as a reliability measure of the metabolite concentration estimates. Higher concentration metabolites (Glu, NAA, Ins, Tau, PCr, Cr) with Cramer-Rao lower bounds (CRLB) under 10\% were considered to be reliably quantified whereas for Glc, GABA, Lac, Asp CRLB under 30\% were considered acceptable and were kept for further analysis.

Neurochemical profiles in thalamus and S1BF of the same rat were acquired serially for 12 rats starting with the thalamic nuclei and S1BF at rest followed by successive 32 -min TGN stimulation periods for S1BF and thalamus. A 15-min period of time was left before acquiring in S1BF at rest, which was used to tilt the holder and perform further adjustments (morphological T2 imaging and Shim) as well as to return the holder to initial position between acquisitions in S1BF and thalamus. A one-way ANOVA test with Bonferroni correction was used to compare metabolite concentrations at rest and during stimulation. The significance level was set at 0.05 . All the results are presented as Mean \pm
SEM. A two-way ANOVA test with Bonferroni correction was performed to compare Glu, GABA, Lac, Glc, and Gln between thalamus and $\mathrm{S} 1 \mathrm{BF}$ brain structures during $\mathrm{ON}$ (stimulation) and OFF (rest) measurement times.

\section{BOLD fMRI}

BOLD responses in the barrel cortex and thalamus were measured post-fMRS measurements using single shot gradient echo EPI in 15 rats ( 7 extra rats underwent BOLD fMRI only, a total of 22 rats underwent BOLD-fMRI). First and second order shims were adjusted using FAST(EST)MAP (Gruetter and Tkác, 2000) resulting in water linewidths of $12 \pm 3 \mathrm{~Hz}$ in a $216 \mu \mathrm{l}(6 \times$ $8 \times 4.5 \mathrm{~mm}^{3}$ ) volume. After echo re-alignment using a reference scan, BOLD responses were assessed using single shot gradient echo EPI $(\mathrm{TR} / \mathrm{TE}=2500-2000 / 25 \mathrm{~ms} ; \mathrm{FOV}=20 \times 20 \mathrm{~mm}$; matrix $=64 \times 64$; slice thickness $=1 \mathrm{~mm} ; 8$ slices, Bandwidth $=325 \mathrm{KHz}, 900$ volumes).

\section{Data Analysis}

Images were analyzed with SPM8 (Matlab; The Mathworks; Natick, USA; Statistical Parametric Mapping, www.fil.ion.ucl. ac.uk/spm/) using the general Linear model (GLM) analyzing each voxel independently and creating a parametric map of statistical significance (Friston et al., 1995). Gradient echo (GRE-EPI) time series were realigned, motion corrected, slice time corrected, normalized to each rat's anatomical images and spatially smoothed with a $3 \mathrm{D}$ Gaussian kernel $(0.6 \times 0.6 \times 1$ $\mathrm{mm}^{3}$ ). Within each analysis, the mean global intensities were mean scaled to an arbitrary value calculated within SPM8. The design model tested was a comparison between "off" and "on" conditions within each TGN stimulation paradigm. The paradigm was convolved with SPM's haemodynamic response function defined as a gamma-variate function and high passfiltered. Residuals for the realigned rat movement were taken into account by submitting the realignment parameters (translations and rotations) as regressors. T-maps were calculated on a voxel by voxel basis. Thresholding criteria of 5 adjacent voxels, each with a T-score $>3.0$ were used to identify regions of interest. Only clusters comprising at least 5 voxels were considered significant ( $p<0.0001$, corrected for multiple comparisons.

With STIMULATE (University of Minnesota, Minneapolis, USA) (Strupp, 1996), regions of interest (ROIs) over the activated primary somatosensory barrel field cortex (S1BF) were drawn with respect to the Paxinos and Watson's Atlas (Paxinos and Watson, 1998). ROIs were delineated from the thresholded tmap of each rat and had the same size as VOIs for $1 \mathrm{H}$-MRS. A representative average time-course was recorded for each animal. When needed baseline correction was performed. T-maps were overlaid on single shot gradient echo EPI images.

\section{Correlations, Robustness, and Statistical Analysis}

Correlations were performed between BOLD responses in thalamus and S1BF, and [Glu] and [GABA] measured at rest and during stimulation in thalamus and S1BF. In addition BOLD responses were also correlated to changes in [Glu] and in [Lac] in S1BF only. Only metabolites with Cramer-Rao lower bounds 


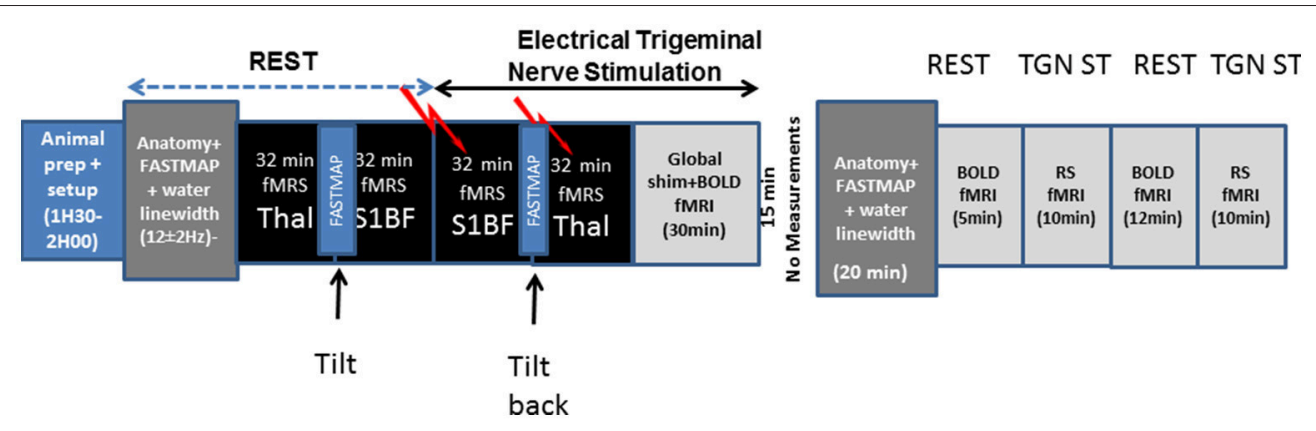

FIGURE 1 | Schematical description of the experimental setup used in the present study and timings.

(CRLB) under 30\% were used for statistical analysis. To examine the association between BOLD responses and metabolites and between metabolites, the Shapiro-wilk test in SPSS 22 was performed for all the variables demonstrating $p$-values above 0.05 and therefore normality of each variable distribution.

The correlation analysis was performed within Origin (OriginLab version 9, Massachusetts, USA). Data were tabulated and scatter plots were obtained. Subsequently, a linear regression analysis was performed allowing to calculate the Pearson's $r$ coefficient of correlation. Linear regression was peformed concomitantly with an ANOVA test with a threshold defined as 0.05 . However, appropriate statistical analysis is required to perform multiple comparisons. With the conservative Bonferroni correction (Bretz et al., 2011) one would assume that all variables were independent which was not necessarily the case. Using a principal component analysis method within Origin, the number of effective comparisons (or eigenvalues) was determined as decribed by Cheverud et al. (1983). The adjusted threshold after Bonferroni correction was therefore $p=\alpha / 4$ with $\alpha=0.05$. Uncorrected $p$-values were denoted as $P$ and were reported as correlating positively or negatively for $P<0.05$. These values were comparable to values reported in the literature for a similar sample size (Table 3).

In order to examine the robustness of the correlation analysis performed in the present study, we further performed a Spearman- Rho correlation analysis within SPSS 22 since for small sample size data sets, a permulation analysis is automatically performed (Bretz et al., 2011). Finally, multiple linear regression analyses were performed in SPSS 22 for a sample size of 15 animals and for a sample size of 22 animals where the animal number was increased by adding 7 animals with CRLBs below $40 \%$ for Glu and GABA, since this type of regression analysis is valid for sample sizes of at least 20 subjects. Using a Kolmonorov-Smirnov test, the multivariate normality was checked again. To avoid over-fitting, a stepwise model analysis was used together with $\mathrm{F}$ statistics. The validity of the regression analysis was assessed and a Durbin-Watson test was included to check for autocorrelation and included a colinearity diagnostic. Homoscedasticity and normality of residuals were tested using standardized plots. To test the robustness of the multiple linear regression analysis Cook's and leverage tests were performed.

\section{RESULTS}

\section{Metabolic Responses to Prolonged TGN Stimulations}

The neurochemical profiles of the thalamus and the barrel cortex before and during TGN stimulation were obtained successively for each rat. High spectra SNR levels were reproducibly measured in S1BF $(91 \pm 8)$ and in the thalamus $(62 \pm 6)$.GABA, Glutamate, Glutamine, Glucose, and Lactate levels were measured at rest and during stimulation periods both in the thalamus $(n=12)$ and in S1BF $(n=15)$ in VOI represented in Figure 2A. In order to increase SNR levels for thalamic MRS acquisitions, the thalamic voxel encompassed several thalamic nuclei of interest [VPM, Reticular Thalamic nucleus (RTN), and Posteromedian Thalamic Nucleus (POm) that can be recognized by reference to the labeled map. Examples of labeled spectra (mean spectra averaged over rat population and over time)] in both structures acquired during stimulation and rest periods are shown (Figure 2B). Increased and decreased levels of Glu and Lac levels during TGN stimulations can be visualized in each structure respectively. Figures 2C,D illustrate positive correlations between BOLD changes and the percent change in amplitude of NAA and $\mathrm{PCr}+$ $\mathrm{Cr}$ peaks respectively. As a consequence of BOLD effect, the linewidths of these peaks should decrease while the peak height should increase. No relationship was found in the thalamus. The neurochemical profiles ( \pm standard error of the mean) are depicted (Figure 3) for each structure and each condition. In the present work, Glutamate and GABA concentrations were compared as well as Glucose and Glutamine in 15 rats (1 rat demonstrated lipid contamination during stimulation and was discarded from further analysis). In the barrel cortex, Lac, Glu, GABA levels were significantly higher during stimulation $(p=0.0015, p=0.015$, and $p=0.021$ respectively, one-way ANOVA and Bonferroni correction). In the thalamus, Gln was significantly higher during TGN stimulation ( $p<0.009$, one-way ANOVA and Bonferroni correction). Although not significantly due to high variability across the rat population, glucose levels decreased in S1BF and thalamus. Regional differences were demonstrated between thalamus and S1BF metabolites at rest and during stimulation using a two-way ANOVA test with Bonferroni correction. Significant differences between brain structures were found for Glu $(p<0.0001)$, Lac $(p=0.02)$, and GABA $(p=$ 
A

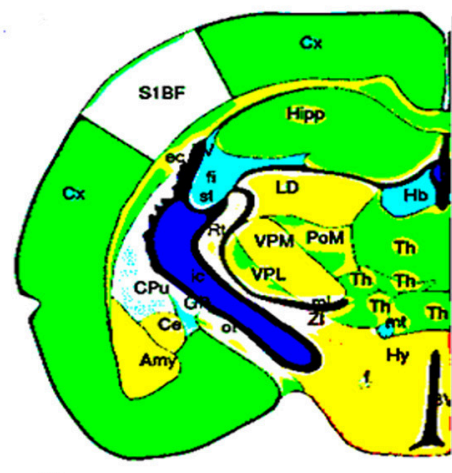

C

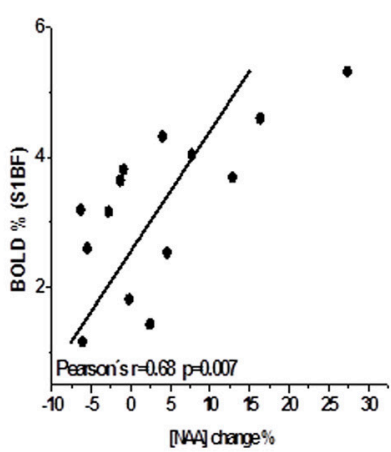

B
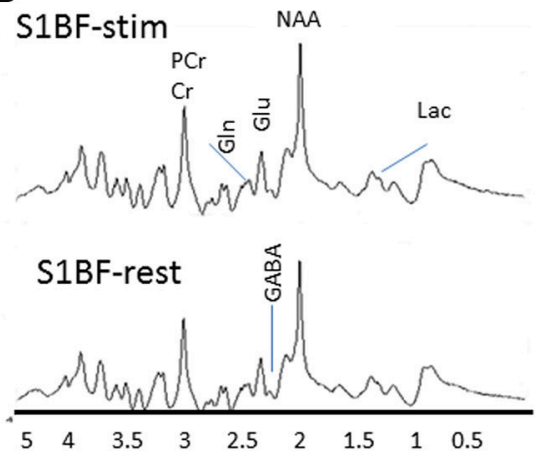

D

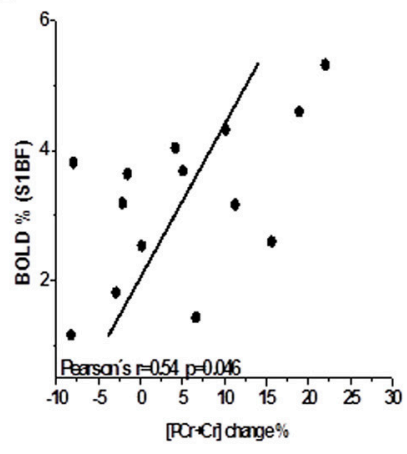

Thal-stim
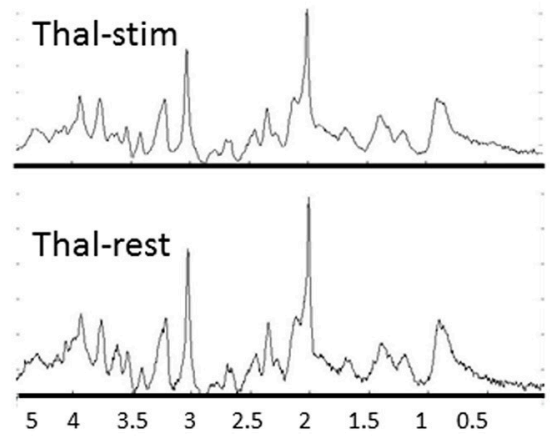

Chemical shift (ppm)

FIGURE 2 | Proton MR Spectroscopy of the rat barrel cortex and the thalamus. (A) BOLD T map overlaid over gradient echo EPI and showing activations both in the barrel cortex and the thalamus. GABA, Glutamate, Glutamine, Glucose, and Lactate levels were measured at rest and during long stimulation periods both in the thalamus $(n=11)$ and in S1BF $(n=15)$ in the represented voxels of interest. The thalamic voxel of interest (36 $\mu$ l) for fMRS in rats is depicted. In order to increase SNR levels for thalamic MRS acquisitions, the thalamic voxel encompassed several thalamic nuclei of interest (VPM, Reticular thalamic nucleus (RTN) and Posteromedian thalamic nucleus (POm)] that can be recognized by reference to the labeled map. Cx, Cortex; Th, Thalamus; S1BF, Primary somatosensory barrel field; Hb, Habenula; Hip, Hippocampus; Cpu, Caudate Putamen; Amy, Amygdala; (B) Examples of labeled spectra (mean spectra averaged over rat population and over time) in both structures acquired during stimulation and rest periods are shown. Increased and decreased levels of Glu and Lac levels during TGN stimulations can be visualized in each structure respectively. (C) and (D) BOLD effects result in a decrease of NAA and tCr (PCr+Cr) peak linewidths (increase in T2*). Changes in linewidths can also be reflected by a change in peak height reported here. Positive correlations $(r=0.68, p=0.007$ and $R=0.54, P=0.046)$ were found between BOLD responses in S1BF and percent change of NAA and $\mathrm{PCr}+\mathrm{Cr}$ peak height respectively. These changes may represent surrogate markers of BOLD responses but remain to be validated.

$0.005)$ during rest and stimulation periods. Changes in [Glu] and [GABA] in both S1BF and thalamus were significantly related to changes related to activation $(p=0.02)$.

\section{Relationships between Bold Responses, Glutamate and GABA in S1BF, and \\ Thalamus}

Although rats underwent sequential prolonged MRS measurements in S1BF and thalamus successively during rest and stimulation periods, BOLD responses were still detected in S1BF and in the thalamus respectively. Unexpectedly, a negative correlation (Pearson's $r=-0.68, p=0.0024$ ) was found as shown in Figure $\mathbf{4 A}$, between BOLD responses in S1BF and thalamus. In order to investigate further this relationship, correlations between BOLD responses, [Glu] and [GABA] were performed within each structure and between structures. BOLD changes in the barrel cortex were negatively correlated to resting GABA levels (Pearson's coefficient $=-0.72, p=0.003$ )
(Figure 4B). Resting Glu levels correlated positively with BOLD changes (Pearson's $r=0.72 ; P<0.03$, Figure 4C) and changes in glutamate $(\triangle \mathrm{Glu})$ were negatively correlated to BOLD (Pearson's coefficient $=-0.70 ; P=0.024$ ) (Figure 4D). A trend toward positive correlation between BOLD changes and Lac changes ( $\triangle \mathrm{Lac}$ ) was also found but not significant (Pearson's $r=0.47$; $P<0.08$ ) (Figure 4E).

In the thalamus, resting or stimulated GABA levels were not correlated to thalamic BOLD responses. Resting S1BF GABA levels demonstrated a positive interaction with thalamic BOLD (Pearson's $r=0.67$ and $P=0.016$, Figure 4F). Resting thalamic Glu levels were not correlated with thalamic BOLD changes (Pearson's $r=0.57 ; P<0.09$ ) and with BOLD changes in the barrel cortex (Pearson's $r=-0.55, P=0.09$ ). A negative correlation between S1BF BOLD and [Glu] during stimulation was also suggested (Pearson's $r=0.62$ and $P=0.055$, Figure 4G).

Since the metabolic status of a structure may be predictive of its functional status including connectivity (Kapogiannis et al., 2013; Castro-Alamancos and Gulati, 2014), the relationships 


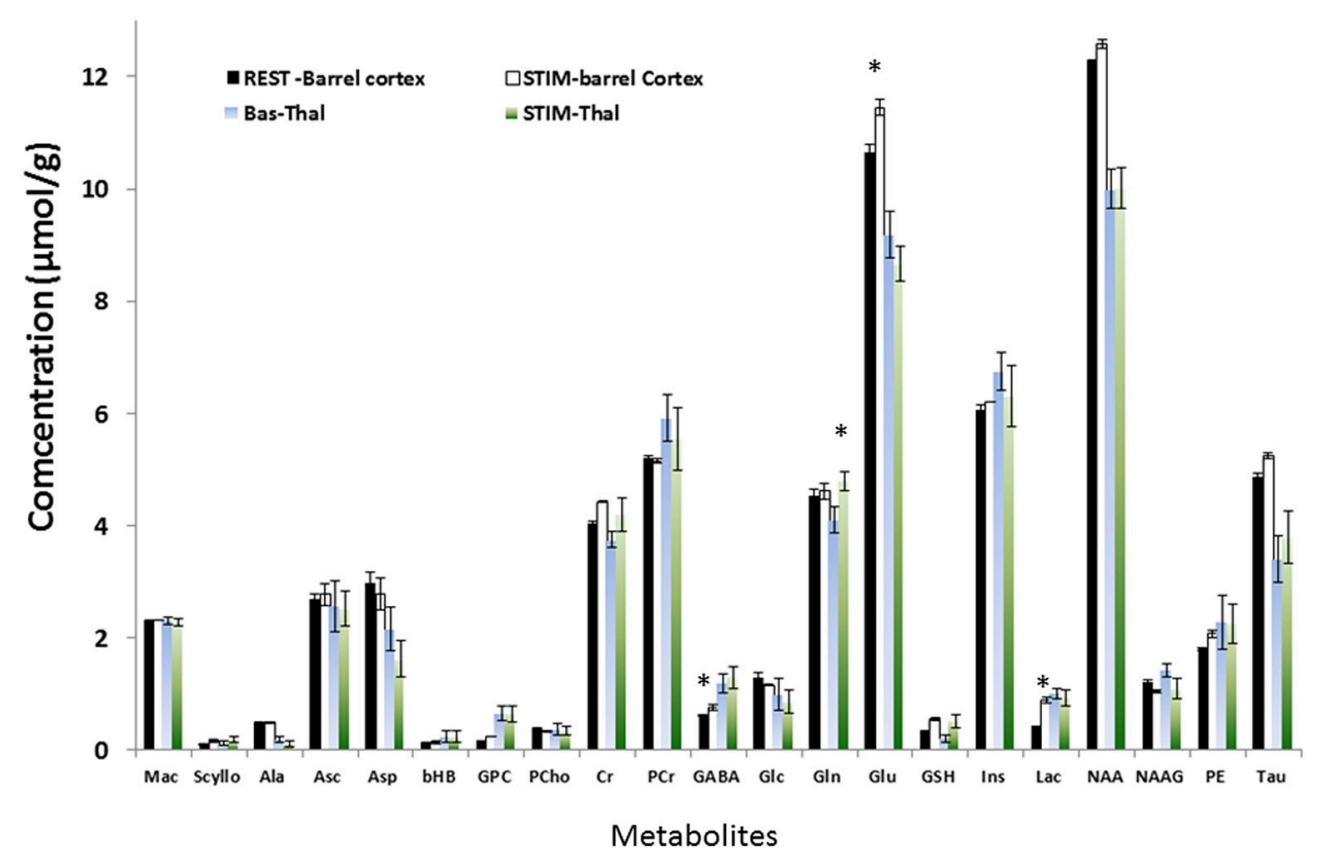

FIGURE 3 | Neurochemical profile of S1BF and thalamus during rest and TGN stimulation for 21 metabolites. In S1BF, Lac, Glu, GABA levels were significantly higher during stimulation ( $p=0.0015, p=0.015$, and $p=0.021$ respectively, one-way ANOVA and Bonferroni correction). Glc and Asp demonstrated a tendency to decrease $(p>0.05)$. In Thalamus, Gln was significantly higher during TGN stimulation $(p<0.009$, ANOVA +Bonferroni) while Glu only showed a strong tendency to decrease. Regional differences were demonstrated between thalamus and S1BF metabolites at rest and during stimulation using a two-way ANOVA test with Bonferroni correction. Significant differences between brain structures were found for Glu $(p<0.0001)$, Lac $(p=0.02)$, and GABA ( $p=0.005)$. Changes in [Glu] and $[\mathrm{GABA}]$ in both $\mathrm{S} 1 \mathrm{BF}$ and thalamus were significantly related to changes related to activation $(p=0.02)$.

between [Glu] and [GABA] within and between structures were also investigated. As the energetic status of the whole brain is tightly controlled at rest, changes due to neuronal activity also involve changes between related structures. As reported in Table 1, during TGN stimulation, there was no correlation between [GABA] and [Glu] whereas at rest, GABA and Glu levels in the barrel cortex were positively correlated (Pearson's $r=$ 0.74; $P=0.014$, Figure 5A) and resting and stimulated [Glu] in $\mathrm{S} 1 \mathrm{BF}$ were positively correlated (Figure $5 \mathrm{~B}$ ). On the other hand, resting thalamic GABA levels were negatively correlated to barrel cortex GABA levels measured during stimulation (Pearson's $r$ $=-0.69 ; P=0.024$ ) (Figure 5D) but not at rest (Figure 5C). However, Glu levels in S1BF at rest strongly influenced stimulated thalamic GABA levels (Figure 5D; Table 1). In S1BF, resting and activated Glu levels were correlated (Pearson's $r=0.67 ; P<0.05$, Figure 5E).

\section{Multiple Linear Regression Analysis and Robustness of the Analysis}

Table 2 presents the results of the Spearman-Rho correlation analysis. Results were in agreement with previous Pearson's correlation ( $p$-values under 0.01 and 0.05 ).

Stepwise non-parameteric multiple linear regression analyis in a sample size of 15 animals revealed that resting Glutamate and GABA levels added significant explanatory power to the regression model. In addition, resting GABA and glutamate levels in the barrel cortex (BC) explained between 52.3 and $89 \%$ of the variance. The Durbin-watson test $(\mathrm{D}=1.631)$ allowed to assume that there was no significant first order linear autocorrelation in the multiplelinear regression data. The F-Test is the test of significance of the multiple linear regression. The $F$-test of was highly significant $\left(F=19.063, p=0.012\right.$ for $\mathrm{Glu}_{\text {rest }}$ and $p=$ 0.001 for $\mathrm{GABA}_{\text {rest }}$,) thus it can be assumed that there is a linear relationship between the variables in our model. Since we have multiple independent variables in the analysis the Beta weights compared the relative importance of each independent variable in standardized terms. We found that a regression model encompassing BOLD and resting Glutamate and GABA levels had the highest impact on the regression $(\beta=0.955$ and 0.383$)$. Multicollinearity in our multiple linear regression model was higher than $\mathrm{MC}=0.811$ showing that there was no suspiscion of multicollinearity. Q-Q plots indicated that in our multiple linear regression analysis there was no tendency in the error terms. Finally, Cook's and centered leverage mean values were 0.223 \pm 0.4 and $0.273 \pm 0.2$ showing that exclusion of data points wouldn't have changed the regression statistics substantially while the centered leverage value demonstrated that there was no significant influence of specific datapoints. The same analysis was performed with a sample size of 22 animals showing that resting glutamate levels only added significant explanatory power to the regression model explaining $80 \%$ of the variance confirmed by highest Beta weights $(\beta=0.896)$. Again, there were no significant influence of autocorrelations and multicollinearity $(\mathrm{D}=2.3, \mathrm{MC}$ $=0.655)$. F-tests were again highly significant $(F=44.837, p<$ 

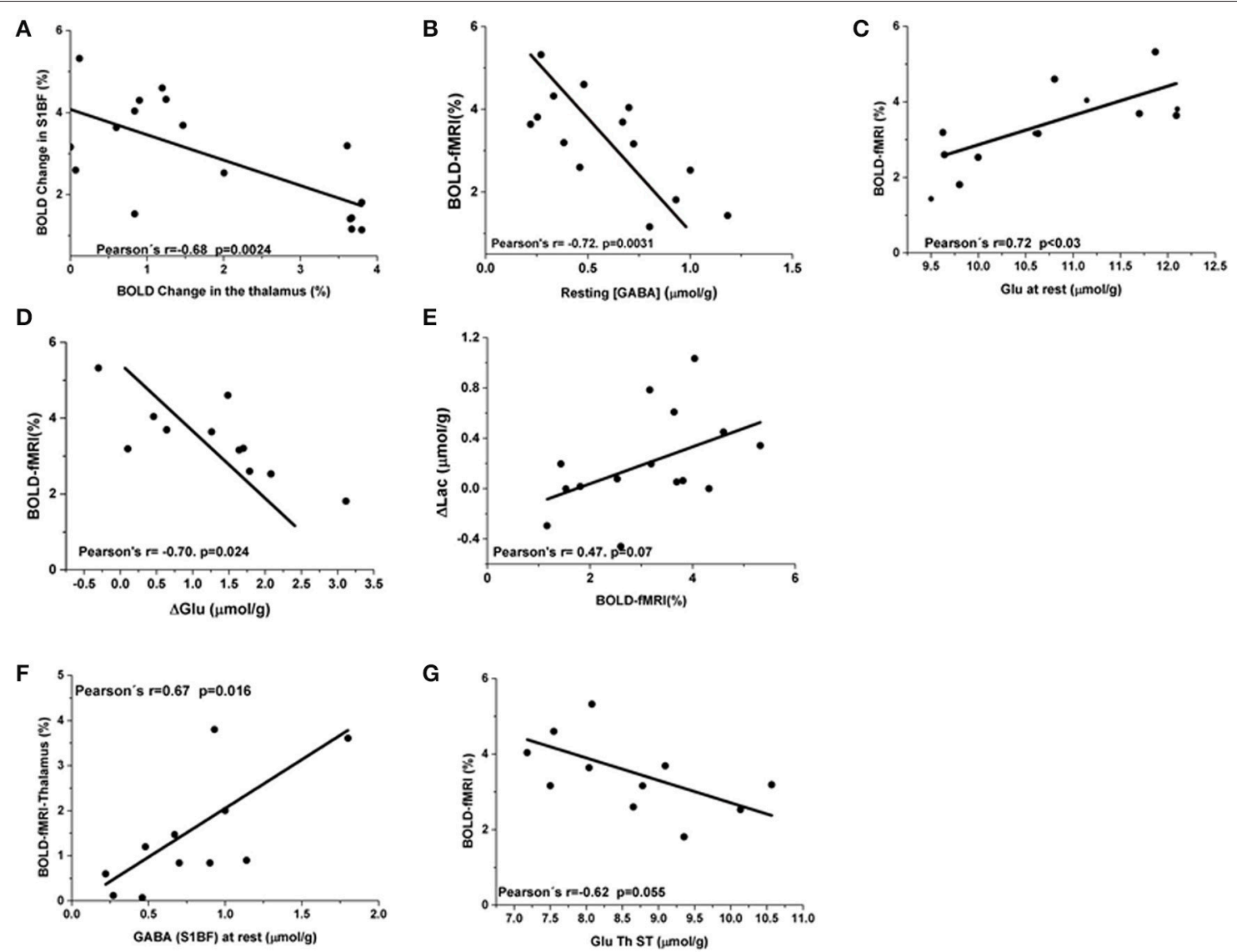

FIGURE 4 | BOLD responses correlations to [GIu] and [GABA] in S1BF. (A) Negative correlation of Blood Oxygen Level dependent percent changes between the barrel cortex and the thalamus (Pearson's $r=-0.68 ; p=0.0024$ ). (B) BOLD changes in the barrel cortex were negatively correlated to resting GABA levels (Pearson's coefficient $=-0.72, p=0.003$ ). (C) A positive correlation between Glu levels at rest and BOLD responses was observed (Pearson's coefficient $=0.72$, $P<0.03$ ). (D) $\triangle$ Glu were negatively correlated to $\triangle B O L D$ (Pearson's coefficient $=-0.70$. $P=0.024$ ). (E) A trend toward positive correlation between BOLD changes and Lac changes was also found (Pearson's $r=0.47 ; P=0.07$ ). Thalamic and S1BF BOLD responses correlations to [Glu] and [GABA]. In the thalamus, GABA levels were not correlated to thalamic BOLD responses neither at rest nor during activation. (F) At rest S1BF GABA levels demonstrated a positive interaction with thalamic BOLD approaching significance (Pearson's $r=0.67$ and $P=0.02$ ). (G) A negative correlation between S1BF BOLD and [Glu] during stimulation also approached significance (Pearson's $r=0.62$ and $P=0.055)$.

0.0001). Finally due to the slight increase in sample size, Cook\#s and centered leverage values were further decreased $0.077 \pm$ 0.258 and $0.048 \pm 0.045$.

\section{DISCUSSION}

In the present work, using fMRS and BOLD fMRI measurements in the thalamus and barrel cortex of rats, the modulation of neuronal activity- represented by BOLD responses- within and between these connected structures by GABA and Glu neurotransmitters was addressed with correlations. To the best of our knowledge, the present study is the first attempt to measure and compare functional hemodynamic and metabolic responses upon sensory stimulation of related brain structures in rats using MR-based methods. Although as discussed below, a number of technical challenges remain to be solved to validate the methodology proposed, the approach is potentially powerful for multi-regional investigations during brain activity and a better understanding of the underlying relationships between neurotransmitters and functional neuroimaging signals as well as functional connectivity.

\section{Confirmation of Cortical BOLD-Neurotransmitter Relations in the Activated Region}

Our study confirmed the cortical BOLD responses dependence on resting GABA levels as well as changes in Glu and Lac in response to stimulation but revealed that the direction of the correlations was specific to the system under analysis. In agreement with previous results obtained in the visual human cortex (Northoff et al., 2007; Donahue et al., 2010; Muthukumaraswamy et al., 2012; Bednařík et al., 2015), positive S1BF BOLD signals were negatively correlated to baseline S1BF GABA levels. In the human visual cortex, concomitant positive, and negative correlations with Cerebral Blood Flow (CBF) and 
TABLE 1 | Glutamate and GABA concentrations correlations in S1BF and Thalamus at rest and during TGN Stimulations.

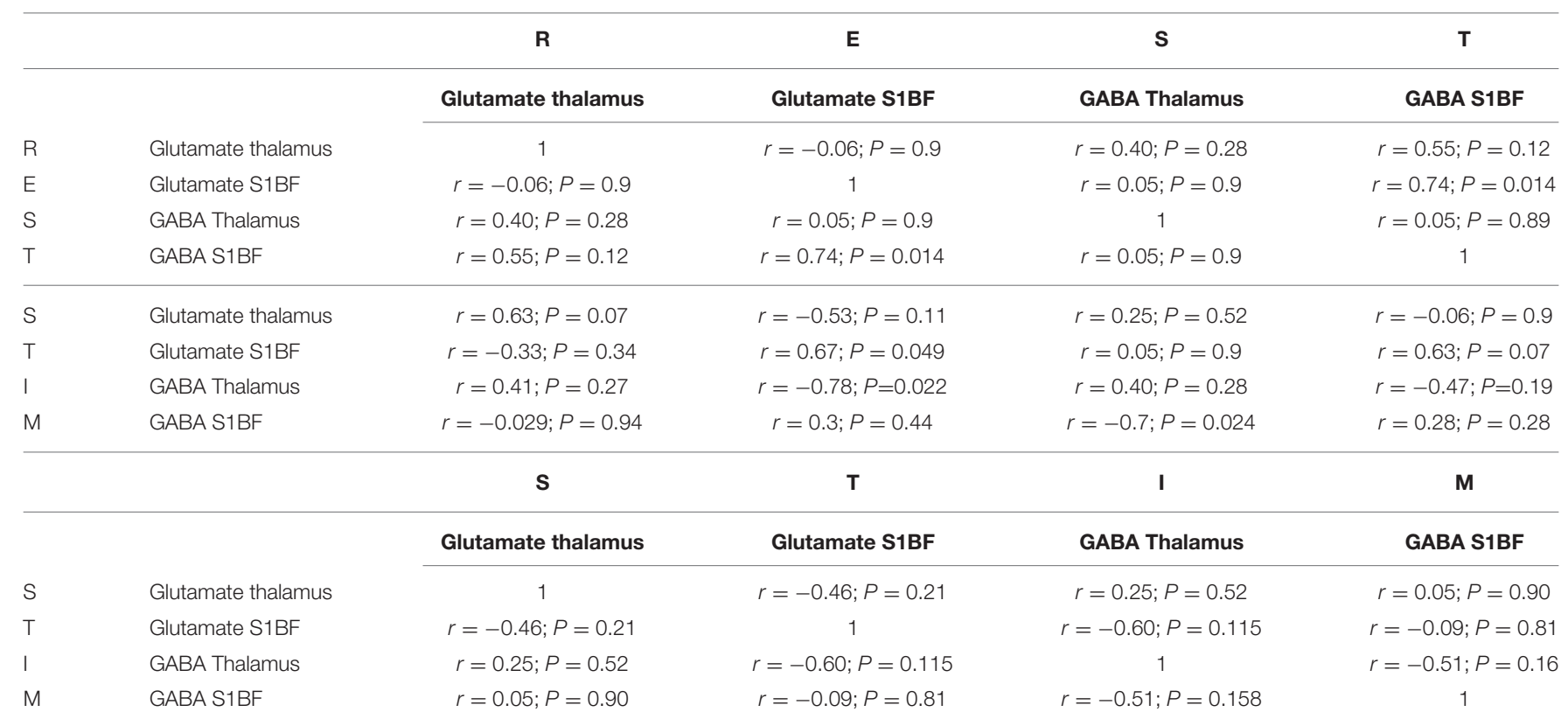
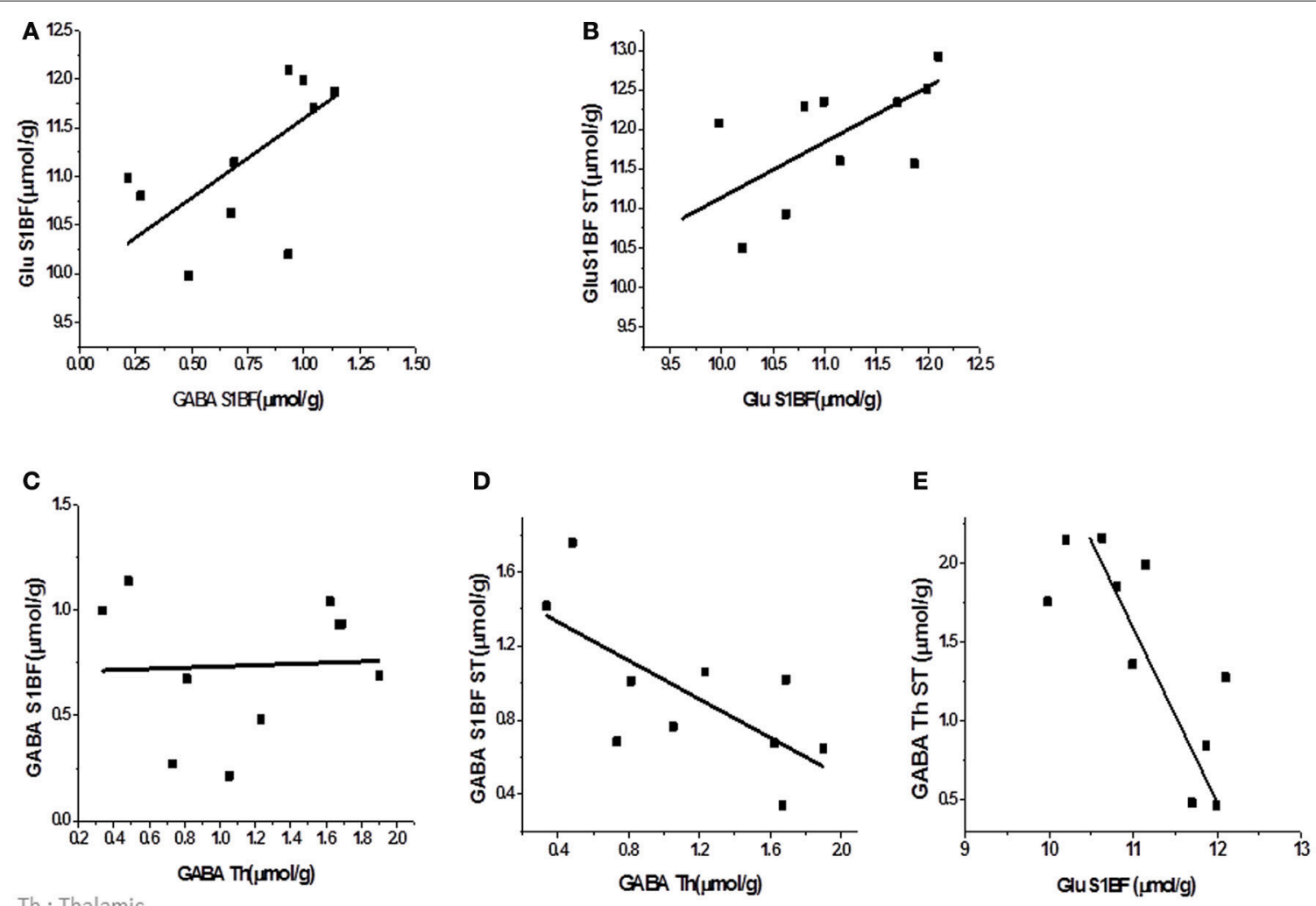

Th: Thalamic

ST: during TGN stimulation

All metabolites were expressed in $\mu \mathrm{mol} / \mathrm{g}$

FIGURE 5 | Correlations between metabolites within and between the thalamus and the cortex. (A) At rest, GABA and Glu levels in the barrel cortex were positively correlated (Pearson's $r=0.74 ; P=0.014$ ). (B) In S1BF, Glu levels at rest and during stimulation were correlated (Pearson's $r=0.67 ; P<0.05)$ (C,D). Thalamic GABA levels at rest were negatively correlated to barrel cortex GABA levels during stimulation (Pearson's $r=-0.69 ; P=0.024)$ but not at rest (Figure 4 C). (E) Glu levels in S1BF at rest were strongly influenced by stimulated thalamic GABA levels. 
TABLE 2 | Spearman-Rho correlation and statistics (** $p<0.01$; $\left.{ }^{*} p<0.05\right)$.

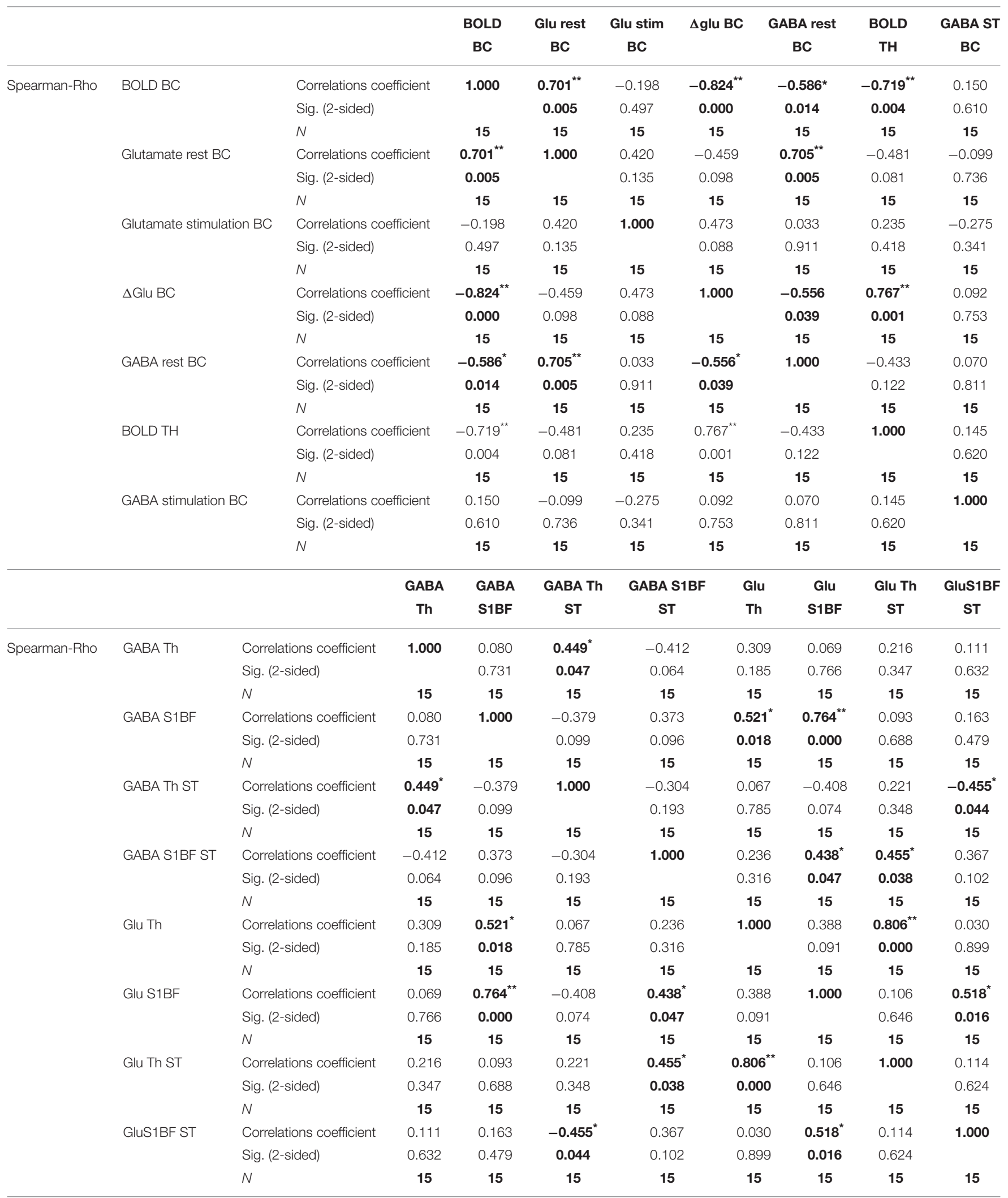

The Spearman-Rho coefficient of correlation between BOLD BC and $\triangle L$ Lac was 0.461 with $p=0.084(n=15)$. 
vascular space occupancy (VASO) signals respectively, were interpreted as a greater influx of blood to the visual cortex region in participants with higher GABA levels in order to fuel the greater amount of excitatory activity required for overcoming the higher resulting inhibition. In S1BF, low $[\mathrm{Glu}]_{\text {rest }}$ predicted lower BOLD responses whereas lower $\Delta[\mathrm{Glu}]$ predicted higher BOLD percent changes during TGN stimulation. Furthermore, $\Delta[\mathrm{Lac}]$ suggested a positive correlation with BOLD fMRI responses (Pearson's $r=0.47 P=0.07$ ). These findings were in line with the study by Bednarík et al. (2015) where higher Glu changes $(\Delta[\mathrm{Glu}])$ as well as higher Lac changes $(\Delta[\mathrm{Lac}])$ predicted higher BOLD percent changes in the human visual cortex, interpreted as a response to increased energy demands of neuronal activation. Although, this study confirmed the existence of relationships between BOLD responses and both GABA at rest and Glu changes, the negative relationship with the latter was unexpected and difficult to interpret: If higher $\Delta$ Glu levels not compensating for the inherently higher GABA levels in S1BF of some rats and therefore leading to lower BOLD responses could represent a reasonable interpretation, null $\Delta$ Glu (at intercept) resulting in highest BOLD responses appeared contradictory to previous results. To date, the majority of fMRS studies reported weak mean $\Delta[\mathrm{Glu}]$ ranging between 2 and $4 \%$ due to cortical stimulation (Mangia et al., 2007; Lin et al., 2012; Schaller et al., 2014). In these studies, fMRS was conducted after carefully positioning the VOI over the core activated area in BOLD maps for which fMRI acquisitions were optimized. In the present study, BOLD responses were measured after fMRS acquisitions. The position of the VOI in S1BF relied on previous work (Just et al., 2013) possibly missing the S1BF activated core and thus shifting the amplitudes of vascular and metabolic responses. However, positive changes in amplitudes of NAA and $\mathrm{tCr}$ peaks as a consequence of BOLD changes were detected as in Just et al. (2013) suggesting that the choice of VOI was adequate. Moreover, averaging the voxels yielding the highest statistically significant S1BF BOLD responses also revealed a negative correlation (not shown) with $\Delta[\mathrm{Glu}]$ thus discarding effects related to thresholding. Weak changes in [Gln] in S1BF also corresponded to rats with highest BOLD responses and could not serve as an explanation. Bednař́k et al. (2015) also found a non-zero $\Delta[\mathrm{Glu}]$ intercept for $\mathrm{BOLD}=0 \%$. Although they point at different sensitivities of fMRI and fMRS signals and agree that larger populations need to be examined, their study and the present one point at other effects. In particular, the effects of other neuromodulators such as norepinephrine, noradrenaline, dopamine etc... also need to be taken into account in future studies and may explain these discrepancies (Fontanez and Porter, 2006; Qiao et al., 2007; Castro-Alamancos and Gulati, 2014). Moreover, the literature reported either positive (Enzi et al., 2012) or negative correlations between BOLD and baseline Glu concentrations, which depended on the health status of patients (Falkenberg et al., 2014) and were mostly inter-regional (Duncan et al., 2014). Therefore, the direction of correlation may be specific to the network under investigation and its physiological status under rest and stimulation. Present results also showed that BOLD changes were strongly dependent on resting state metabolic conditions in S1BF.

\section{Thalamocortical Relationships between BOLD Responses [Glu] and [GABA]}

During TGN stimulation, GABA and Glu levels were uncorrelated within each structure and between structures. On the other hand, resting [Glu] and [GABA] in S1BF were correlated suggesting a strong interplay between these neurotransmitters in S1BF to maintain the EIB at stable levels as would be expected without specific brain activity. Such a relationship was not found in the thalamus and attributed to a lack of specificity since the region of interest taken into account encompassed several thalamic nuclei in order to increase SNR levels for single voxel MR ${ }^{1} \mathrm{H}$ spectroscopy. Notably, the VOI emcompassed the reticular thalamic nucleus described as the only source of thalamic GABAergic inhibition. In addition, it receives inputs from the cerebral cortex but does not project to the cerebral cortex. The inclusion of this nucleus may explain differences between thalamic and cortical neurochemical profiles but also uncorrelated thalamic BOLD responses and resting thalamic GABA levels. Many studies suggested that RTN plays a significant role in thalamic gating, facilitating relevant stimuli and inhibiting others by increased inhibition of the information transfer via other relay nuclei. The shape and size of RTN make this important nucleus very difficult to avoid. Other techniques such as proton MR spectroscopic imaging (MRSI) (Seuwen et al., 2015) may be more adequate for future investigations while fMRS techniques coupled to optogenetic stimulation may help unraveling the exact contribution of this structure (Jurgens et al., 2012).

A significant negative correlation between BOLD responses measured in both S1BF and thalamus was found. In the literature, weak or absent thalamic BOLD responses with regards to forepaw or barrel cortex activations were reported in the past (Esaki et al., 2002; Keilholz et al., 2004; Zhao et al., 2008; Mishra et al., 2011; Devonshire et al., 2012). Thalamic BOLD responses are usually expected to be weaker than cortical ones due to generally higher GABA levels linked to higher densities of GABAergic neurons (Mishra et al., 2011; Devonshire et al., 2012; Tiwari et al., 2013). Here, accordingly, thalamic GABA levels were higher than in $\mathrm{S} 1 \mathrm{BF}$ at rest and during stimulation but there was no significant increase of mean GABA levels in the thalamus upon TGN stimulation. Moreover, thalamic BOLD responses and GABA levels were uncorrelated. While these findings may be attributed to the inclusion of RTN, rats with higher resting S1BF GABA levels had high amplitude BOLD responses in the thalamus correlated to low amplitude $\mathrm{BOLD}$ responses in S1BF. Moreover, $[\mathrm{Glu}]_{\text {rest }}$ in S1BF were negatively correlated to $[\mathrm{GABA}]_{\text {stim }}$ in the thalamus. Therefore, rats with low Glu levels and high resting GABA levels in S1BF demonstrated lower BOLD responses in S1BF. Consequently, the release of high GABA concentrations in the thalamus could be interpreted as a way to overcome the higher excitatory activity supported by significantly increased thalamic Gln levels during 
TGN stimulation and thus balancing the decreased Glu levels and inducing strong BOLD thalamic activation. In the thalamus, [Gln] increased significantly during TGN stimulation while [Glu] decreased without significance. Interestingly, identical changes were reported by $\mathrm{Xu}$ et al. (2005) in the forepaw cortex of rats and were attributed to an augmented release of Glu and a rapid conversion into Gln through the Glu-Gln cycle between glutamatergic neurons and astrocytes. (Hirata and CastroAlamancos, 2010) suggested that neuromodulators can control the synaptic activity of thalamocortical and corticothalamic cells and therefore control the state of their respective targets without being released there directly, thereby ensuring that both structures are under specific conditions essential for a proper connectivity. This is furthermore supported by studies suggesting the control of the synchrony of neuronal activity by regional EIB (Kapogiannis et al., 2013) and its correlation with functional connectivity. Therefore, the present study confirmed the interest in investigating relationships between neurotransmitters within the thalamus and the barrel cortex and between these two regions as potential predictors of functional connectivity, which could be of interest for studying pathologies such as absence epilepsy or schizophrenia. Duncan et al. (2014) in their review pointed at the fact that most studies looking at the roles of GABA and glutamate in brain function remain at the correlational stage while most of them include small numbers of subjects thus limiting the validation of findings. In the present work, functional connectivity was conducted on 8 rats only and showed high variability across this population (Figures S1, S2). At this stage, findings remain only indicative and should be further validated with more robust resting-state connectivity analysis. Figure 6 illustrates a preliminary schematical overview of the Glutamate and GABA modulatory effects and functional connectivity on cortico-thalamo cortical loop in a healthy rat.

\section{LIMITATIONS AND PERSPECTIVES}

\section{Statistical Analysis and Multiple Comparisons}

In the text, correlations between either BOLD responses and metabolites or between metabolites were reported as being significant for uncorrected $p$-values. Upon application of corrected $p$-values as stated in Materials and Methods most correlations did not pass significance level which was attributed to the small number of animals included in the study. As a consequence of this, the correlations reported here point at interesting effects that remain to be validated. Nevertheless, to date, most of the correlation studies involving BOLD-metabolite relationships were not corrected for multiple comparisons. Table 3 summarizes various results from these studies with Pearson's correlation coefficients, $\mathrm{p}$ values and subject numbers. The column "statistics" mentions the statical test applied to the correlation. Unfortunately, these studies were mainly performed in humans. The number of subjects taken into account was in general similar to the number of rats used in the present study while significant negative correlation of brain activity measures with resting GABA were consistently

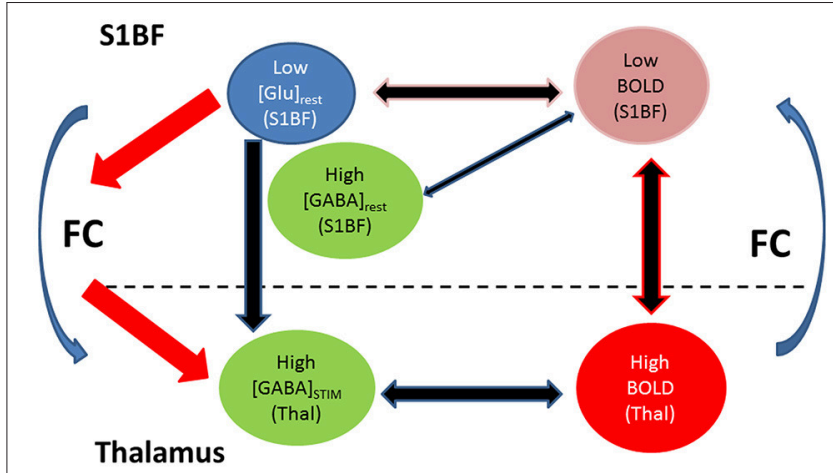

FIGURE 6 | Preliminary schematical overview of the Glutamate and GABA modulatory effects and functional connectivity on cortico-thalamo cortical loop in a healthy rat with low BOLD in S1BF $(1-2 \%)$ under prolonged trigeminal nerve electrical stimulation. Multi-regional investigations during brain activity may help understanding how neurotransmitters relate to functional neuroimaging signals and functional connectivity. Within each structure BOLD responses were modulated by within structure GABA and/or Glutamate levels. In the thalamus, BOLD responses were modulated by GABA during stimulation. These thalamic GABA levels demonstrated strong dependence on resting Glu levels in S1BF. Glutamatergic and GABAergic processes modulate thalamocortical and corticothalamic functional connectivity $(\mathrm{FC})$. We postulate that these processes may involve regulation of neuronal synchrony between thalamus and cortex.

found across studies including this one but not correlations with Glu levels. Although significance levels were not always adjusted, all these studies demonstrate that BOLD variability can be explained by GABA levels at least in cortical areas and the negative relationship between BOLD-resting GABA is a hallmark of neuronal activity. Here, the robustness of the correlation study was further confirmed by the Spearman-Rho correlation analysis and the multiple linear regression analysis. Although we delibaretely added Glutamate and GABA levels estimates with lower reliability (CRLB $<=40 \%$ ) from seven animals, we found that $\mathrm{F}$ statistics were significant while calculation of distance values (Cook's and leverage) increased the robustness of the analysis compared to a lower sample size of 15 animals. In addition, these analyses emphasized the roles of S1BF resting glutamate and GABA levels. The multiple linear regression analysis further demonstrated the need for higher sample sizes to validate metabolic correlations in rats but also pointed at the need for improved methods for associating MRS values as the interpretation of multiple linear regression models with an increased number of variables becomes extremely complicated.

\section{Study Design}

One of the main pitfalls of the present study was its sequential design that unfortunately did not allow simultaneous assessment of BOLD, thalamic and S1BF metabolites. Using magnetic MRSI techniques such as FIDLOVs (Seuwen et al., 2015) which would at the same time contribute to preserve SNR levels and time may help while there is definitely a need for increased targeting at smaller structures with higher SNR levels for an increased specificity of studies as mentioned earlier for RTN or in pathologies (Pan et al., 2015). Finally, BOLD responses 
TABLE 3 | BOLD-metabolites relationships in various human studies.

\begin{tabular}{|c|c|c|c|c|c|c|}
\hline Study & Brain area & Correlation & Pearson's R & $P$-value & Number of participants & Statistics \\
\hline \multirow[t]{4}{*}{ Bednařík et al., 2015} & Visual cortex & $\begin{array}{l}\text { BOLD vs. water } \\
\text { linewidth change }\end{array}$ & 0.93 & 0.0001 & 11(15) & Not explained \\
\hline & & BOLD vs. $\Delta$ Glu & 0.93 & 0.001 & 11(15) & Not explained \\
\hline & & BOLD vs. $\Delta$ Lac & 0.65 & 0.003 & 11(15) & Not explained \\
\hline & & BOLD vs. GABA & -0.65 & 0.043 & $11(15)$ & Not explained \\
\hline \multirow[t]{4}{*}{ Donahue et al., 2010} & Visual cortex & BOLD vs. GABA & -0.7 & $P<0.05$ & 12 & $\begin{array}{l}\text { two tailed } \\
P \text {-value }\end{array}$ \\
\hline & & VASO vs.GABA & -0.71 & $P<0.05$ & 12 & \\
\hline & & ASL vs.GABA & 0.41 & $P>0.05$ & 12 & \\
\hline & & CBF vs.GABA & 0.65 & $P<0.05$ & 12 & \\
\hline \multirow{2}{*}{$\begin{array}{l}\text { Muthukumaraswamy } \\
\text { et al., } 2012\end{array}$} & Visual cortex & BOLD vs. GABA & -0.64 & $P<0.05$ & $10(12)$ & Not explained \\
\hline & & $\begin{array}{l}\text { BOLD vs. } \gamma \\
\text { frequency }\end{array}$ & -0.87 & $P<0.01$ & 10(12) & Not explained \\
\hline \multirow[t]{2}{*}{ Duncan et al., 2011} & $\begin{array}{l}\text { Supragenual and } \\
\text { anterior }\end{array}$ & $\begin{array}{l}\text { BOLD sgACC vs. } \\
\text { (Glu+Gln in } \\
\text { pgACC) }\end{array}$ & Not reported & Not reported & 12 & $\begin{array}{l}\text { Spearman's } \\
\text { rho, two tailed }\end{array}$ \\
\hline & Cingulate cortex & & & & & \\
\hline \multirow[t]{2}{*}{ Enzi et al., 2012} & $\begin{array}{l}\text { Anterior cingulate } \\
\text { cortex }\end{array}$ & $\begin{array}{l}\text { BOLD vs. } \\
\text { (Glu+Gln) }\end{array}$ & range: -0.436 to 0.853 & $<0.001-0.432$ & $15-17$ & Paired $t$-tests \\
\hline & Left anterior insula & $\begin{array}{l}\text { BOLD vs. } \\
\text { (Glu+Gln) }\end{array}$ & range: -0.357 to 0.09 & $>0.1$ & $16-18$ & \\
\hline \multirow[t]{4}{*}{ Kühn et al., 2016} & $\begin{array}{l}\text { Inferior frontal } \\
\text { gyrus }\end{array}$ & BOLD vs. GABA & $-0.169 ;-0.352$ & $0.152 ; 0.502$ & 18 & Not explained \\
\hline & & BOLD vs. Glu & $0.144 ; 0.172$ & $0.569 ; 0.494$ & 18 & Not explained \\
\hline & $\begin{array}{l}\text { Anterior cingulate } \\
\text { cortex }\end{array}$ & BOLD vs. GABA & $-0.522 ;-0.475$ & $0.026 ; 0.046$ & 18 & Not explained \\
\hline & & BOLD vs. Glu & $-0.27 ;-0.241$ & $0.279 ; 0.346$ & 18 & Not explained \\
\hline \multirow[t]{3}{*}{ Stagg et al., 2011} & Motor cortex & $\begin{array}{l}\text { Cortical excitability } \\
\text { vs. Glu }\end{array}$ & 0.803 & 0.015 & 12 & $\begin{array}{l}\text { Bonferroni } \\
\text { correction }\end{array}$ \\
\hline & & $\begin{array}{l}\text { Cortical excitability } \\
\text { vs. GABA }\end{array}$ & -0.79 & 0.018 & 12 & $\begin{array}{l}\text { Bonferroni } \\
\text { correction }\end{array}$ \\
\hline & & $\begin{array}{l}\text { Glx/cr vs. FC in } \\
\text { PgACC and Al }\end{array}$ & -0.51 (Dep) & 0.031 & & \\
\hline Horn et al., 2010 & pgACC, Al & & 0.15 & 0.49 & $18-22$ & Not explained \\
\hline
\end{tabular}

may be measured directly from fMRS acquisitions using changes in linewidths of NAA or total Creatine peaks as a result of activation which would also decrease scanning time. Again these measurements require high SNR levels per subject and require validation against standard BOLD acquisitions. In order to increase SNR levels for the temporal assessment of metabolite changes during TGN stimulation (not presented here), more fids were acquired increasing significantly the scanning time. Adaptation/habituation effects may therefore have been induced in the present study and will certainly need to be taken into account in future investigations.

\section{Impact of Anesthesia}

In the present study, fMRS findings in the cortex of rats under $\alpha$-chloralose anesthesia were similar to findings obtained in humans (Schaller et al., 2014; Bednařík et al., 2015). BOLDGABA and BOLD- $\triangle \mathrm{Lac}$ correlations had similar directions. Nevertheless, the impact of anesthesia on the modulatory effects of Glu and GABA on thalamic and S1BF BOLD responses as well as resting-state functional connectivity cannot be neglected (Williams et al., 2010).

\section{CONCLUSION}

In rats under $\alpha$-chloralose anesthesia, barrel cortex fMRS findings and association of these findings with BOLD responses were in accordance with recent results obtained in the human visual cortex. These results suggested consistent regulatory roles of both glutamate and GABA on cortical neural activity across species. Within the thalamus, neurochemical profiles during TGN stimulation differed from those obtained in the barrel cortex. The correlation study conducted within and between cortical and subcortical structures suggested a complex interplay between glutamatergic and GABAergic modulations of the cortico-thalamo-cortical loop. In addition, preliminary results suggested regulation of functional connectivity between thalamus and cortex by excitatoryinhibitory neurotransmitters. These results will need to be 
followed up with larger population sizes to demonstrate their validity.

To the best of our knowledge, this is the first study investigating two interconnected brain regions during both rest and activation periods using fMRS in rodents. Although in its early stage, the proposed methodology allowed investigating the different contributions of excitatory and inhibitory neurtransmitters on neuronal activity indirectly measured with BOLD in the thalamus and barrel cortex, which could benefit investigations of diseases affecting thalamo-cortical neurotransmission (Autism, Parkinson's disease, Gilles de la Tourette syndrome, cardiac dysfunction...).

\section{AUTHOR CONTRIBUTIONS}

NJ conceived and designed the project. NJ performed experiments and analyzed the data. NJ wrote the manuscript. SS contributed to the discussion and revised the manuscript.

\section{FUNDING}

This study was supported by the Centre d'Imagerie BioMédicale (CIBM) of Ecole Polytechnique Fédérale de Lausanne (EPFL), the University of Lausanne (UNIL) and the Foundations Leenards et Jeantet. SS was funded by a National Competence Center Biomedical Imaging grant (NCCBI).

\section{REFERENCES}

Bednařík, P., Tkáč, I., Giove, F., DiNuzzo, M., Deelchand, D. K., Emir, U. E., et al. (2015). Neurochemical and BOLD responses during neuronal activation measured in the human visual cortex at 7 Tesla. J. Cereb. Blood Flow Metab. 35, 601-610. doi: 10.1038/jcbfm. 2014.233

Behrendt, R. P. (2006). Dysregulation of thalamic sensory "transmission" in schizophrenia: neurochemical vulnerability to hallucinations. J. Psychopharmacol. 20, 356-372. doi: 10.1177/0269881105057696

Bretz, F., Hothorn, T., and Westfall, P. (2011). Multiple Comparisons Using R. Boca Raton, FL: CRC Press Taylor and Francis Group.

Castro-Alamancos, M. A., and Gulati, T. (2014). Neuromodulators produce distinct activated states in neocortex. J. Neurosci. 34, 12353-12367. doi: 10.1523/JNEUROSCI.1858-14.2014

Chen, Z., Silva, A. C., Yang, J., and Shen, J. (2005). Elevated endogenous GABA level correlates with decreased fMRI signals in the rat brain during acute inhibition of gaba transaminase. J. Neurosci. Res. 79, 383-391. doi: $10.1002 /$ jnr.20364

Cheverud, J., Rutledge, J., and Atchley, W. (1983). Quantitative genetics of development: genetic correlations among age-specific trait values and the evolution of antogeny. Evolution 37, 895-905. doi: 10.2307/2408405

De Groof, G., Jonckers, E., Güntürkün, O., Denolf, P., Van Auderkerke, J., and Van der Linden, A. (2013). Functional MRI and functional connectivity of the visual system of awake pigeons. Behav. Brain Res. 239, 43-50. doi: 10.1016/j.bbr.2012.10.044

Devonshire, I. M., Papadakis, N. G., Port, M., Berwick, J., Kennerley, A. J., Mayhew, J. E., et al. (2012). Neurovascular coupling is brain region-dependent. Neuroimage 59, 1997-2006. doi: 10.1016/j.neuroimage.2011.09.050

Devor, A., Ulbert, I., Dunn, A. K., Narayanan, S. N., Jones, S. R., Andermann, M. L., et al. (2005). Coupling of the cortical hemodynamic response to cortical and thalamic neuronal activity. Proc. Natl. Acad. Sci. U.S.A. 102, 3822-38227. doi: 10.1073/pnas.0407789102

\section{ACKNOWLEDGMENTS}

The author would like to thank Prof. Rolf Gruetter for providing the necessary tools for this study.

\section{SUPPLEMENTARY MATERIAL}

The Supplementary Material for this article can be found online at: http://journal.frontiersin.org/article/10.3389/fphys. 2017.00030/full\#supplementary-material

Figure S1 | Resting-state BOLD fMRI. (A,B) BOLD T map overlaid over gradient echo EPI and showing bilateral resting-state BOLD both in the barrel cortex (A) and the thalamus (B) during long stimulation periods (2 min OFF-10 minON-2 minOFF) and short stimulation periods (30 s OFF-30 s ON...). (C). Correlations between left and right hemisphere time courses for bilateral seeds were increased after long stimulations in individual animals but did not reach significance at the population level.

Figure S2 | Influence of [Glu] and [GABA] during rest and stimulation on cortico-cortical and thalamo-thalamic functional connectivities. (A,B) Highest positive Pearson's correlation coefficients in both contralateral and ipsilateral S1BF ( $r=0.57-0.67)$, (A) were found between stimulated Glu levels and $T$-values while cluster numbers were mainly correlated to GABA levels at rest (B). (C) Thalamo-thalamic FC was negatively correlated to stimulated GABA levels (D-G) At rest, thalamic GABA levels were positively correlated to cluster numbers $(r=+0.57$. (D) A positive relationship between stimulated thalamic Glu levels and S1BF $T$-values $(r=0.69)$. (E) was observed but a negative one was observed between stimulated thalamic GABA levels and both $T$-values and Cluster numbers $(r=-0.57,-0.56$ respectively) $\mathbf{( F , G ) . ~ ( H ) ~ N e g a t i v e ~ c o r r e l a t i o n ~ b e t w e e n ~}$ cortico-thalamic FC and glutamate at rest.

Donahue, M. J., Near, J., Blicher, J. U., and Jezzard, P. (2010). Baseline GABA concentration and fMRI response. Neuroimage 53, 392-398. doi: 10.1016/j.neuroimage.2010.07.017

Duncan, N. W., Enzi, B., Wiebking, C., and Northoff, G. (2011). Involvement of glutamate in rest-stimulus interaction between perigenual and supragenual anterior cingulate cortex: a combined fMRI-MRS study. Hum Brain Mapp. 32, 2172-2182. doi: 10.1002/hbm. 21179

Duncan, N. W., Wiebking, C., and Northoff, G. (2014). Associations of regional GABA and glutamate with intrinsic and extrinsic neural activity in humansa review of multimodal imaging studies. Neurosci. Biobehav. Rev. 47, 36-52. doi: 10.1016/j.neubiorev.2014.07.016

Enager, P., Piilgaard, H., Offenhauser, N., Kocharyan, A., Fernandes, P., Hamel, E., et al. (2009). Pathway-specific variations in neurovascular and neurometabolic coupling in rat primary somatosensory cortex. J. Cereb. Blood Flow Metab. 29, 976-986. doi: 10.1038/jcbfm.2009.23

Enzi, B., Duncan, N. W., Kaufmann, J., Tempelmann, C., Wiebking, C., and Northoff, G. (2012). Glutamate modulates resting state activity in the perigenual anterior cingulate cortex - a combined fMRI-MRS study. Neuroscience 227, 102-109. doi: 10.1016/j.neuroscience.2012.09.039

Esaki, T., Itoh, Y., Shimoji, K., Cook, M., Jehle, J., and Sokoloff, L. (2002). Blockade of $\mathrm{K}_{\mathrm{ATP}}$ channels with glibenclamide does not alter functional activation of cerebral blood flow in the unanesthetized rat. Brain Res. 948, 56-63. doi: 10.1016/S0006-8993(02)02948-7

Falkenberg, L. E., Westerhausen, R., Craven, A. R., Johnsen, E., Kroken, R. A. L., Berg, E. M., et al. (2014) Impact of glutamate levels on neuronal response and cognitive abilities in schizophrenia. Neuroimage Clin. 4, 576-584. doi: 10.1016/j.nicl.2014.03.014

Feldmeyer, D., Brecht, M., Helmchen, F., Petersen, C. C., Poulet, J. F., Staiger, J. F., et al. (2013). Barrel cortex function. Prog. Neurobiol. 103, 3-27. doi: 10.1016/j.pneurobio.2012.11.002

Fontanez, D. E., and Porter, J. T. (2006) Adenosine A1 receptors decrease thalamic excitation of inhibitory and excitatory neurons in the barrel cortex. Neuroscience 137, 1177-1184. doi: 10.1016/j.neuroscience.2005.10.022 
Friston, K. J., Holmes, A. P., Poline, J. B., Grasby, P. J., Williams, S. C., Frackowiak, R. S., et al. (1995). Analysis of fMRI time-series revisited. Neuroimage 2, 45-53. doi: 10.1006/nimg.1995.1007

Gruetter, R., and Tkác, I. (2000). Field mapping without reference scan using asymmetric echo-planar techniques. Magn. Reson. Med. 43, 319-323. doi: 10.1002/(SICI)1522-2594(200002)43:2<319::AID-MRM22>3.0.CO;2-1

Hirata, A., and Castro-Alamancos, M. A. (2010). Neocortex network activation and deactivation states controlled by the thalamus. J. Neurophysiol. 103, 1147-1157. doi: 10.1152/jn.00955.2009

Horn, D. I., Yu, C., Steiner, J., Buchmann, J., Kaufmann, J., Osoba, A., et al. (2010). Glutamatergic and resting-state functional connectivity correlates of severity in major depression - the role of pregenual anterior cingulate cortex and anterior insula. Front. Syst. Neurosci. 4:33. doi: 10.3389/fnsys.2010.00033

Jurgens, C. W., Bell, K. A., McQuiston, A. R., and Guido, W. (2012). Optogenetic stimulation of the corticothalamic pathway affects relay cells and GABAergic neurons differently in the mouse visual thalamus. PLOS ONE 7:e45717. doi: 10.1371/journal.pone.0045717

Just, N., Petersen, C., and Gruetter, R. (2010). BOLD responses to trigeminal nerve stimulation. Magn. Reson. Imaging 28, 1143-1151. doi: 10.1016/j.mri.2010.02.002

Just, N., Xin, L., Frenkel, H., et al. (2013). Characterization of sustained BOLD activation in the rat barrel cortex and neurochemical consequences. Neuroimage 74, 343-351. doi: 10.1016/j.neuroimage.2013.02.042

Kapogiannis, D., Reiter, D. A., Willette, A. A., and Mattson, M. P. (2013). Posteromedial cortex glutamate and GABA predict intrinsic functional connectivity of the default mode network. Neuroimage64, 112-119. doi: 10.1016/j.neuroimage.2012.09.029

Keilholz, S. D., Silva, A. C., Raman, M., Merkle, H., and Koretsky, A. P. (2004) Functional MRI of the rodent somatosensory pathway using multislice echo planar imaging. Magn. Reson. Med. 52, 89-99. doi: 10.1002/mrm.20114

Kocharyan, A., Fernandes, P., Tong, X. K., Vaucher, E., and Hamel, E. (2008) Specific subtypes of cortical GABA interneurons contribute to the neurovascular coupling response to basal forebrain stimulation. J. Cereb. Blood Flow Metab. 28, 221-231. doi: 10.1038/sj.jcbfm.9600558

Kühn, S., Schubert, F., Mekle, R., Wenger, E., Ittermann, B., Lindenberger, U., et al. (2016). Neurotransmitter changes during interference task in anterior cingulate cortex: evidence from fMRI-guided functional MRS at 3 T. Brain Struct. Funct. 221, 2541-2551. doi: 10.1007/s00429-015-1057-0

Lecrux, C., Toussay, X., Kocharyan, A., Fernandes, P., Neupane, S., Lévesque, M., et al. (2011). Pyramidal neurons are "neurogenic hubs" in the neurovascular coupling response to whisker stimulation. J. Neurosci. 31, 9836-9847. doi: 10.1523/JNEUROSCI.4943-10.2011

Lin, Y., Stephenson, M. C., Xin, L., Napolitano, A., and Morris, P. G. (2012). Investigating the metabolic changes due to visual stimulation using functional proton magnetic resonance spectroscopy at 7T. J. Cereb. Blood Flow Metab. 32, 1484-1495. doi: 10.1038/jcbfm.2012.33

Logothetis, N. K., Augath, M., Murayama, Y., Rauch, A., Sultan, F., Goense, J., et al. (2010). The effects of electrical microstimulation on cortical signal propagation. Nat. Neurosci. 13, 1283-1291. doi: 10.1038/nn.2631

Lüttjohann, A., and van Luijtelaar, G. (2015). Dynamics of networks during absence seizure's on- and offset in rodents and man. Front. Physiol. 6:16. doi: $10.3389 /$ fphys.2015.00016

Mangia, S., Tkác, I., Gruetter, R., Van de Moortele, P. F., Maraviglia, B., and Uğurbil, K. (2007). Sustained neuronal activation raises oxidative metabolism to a new steady-state level: evidence from 1H NMR spectroscopy in the human visual cortex. J. Cereb. Blood Flow Metab. 27, 1055-1063. doi: $10.1038 /$ sj.jcbfm. 9600401

Mishra, A. M., Ellens, D. J., Schridde, U., Motelow, J. E., Purcaro, M. J., DeSalvo, M. N., et al. (2011). Where fMRI and electrophysiology agree to disagree: corticothalamic and striatal activity patterns in the WAG/Rij rat. J. Neurosci.31, 15053-15064. doi: 10.1523/JNEUROSCI.0101-11.2011

Mlynárik, V., Gambarota, G., Frenkel, H., and Gruetter, R. (2006). Localized shortecho-time proton MR spectroscopy with full signal-intensity acquisition. Magn. Reson. Med. 56, 965-970. doi: 10.1002/mrm.21043
Muthukumaraswamy, S. D., Evans, C. J., Edden, R. A., Wise, R. G., and Singh, K. D. (2012). Individual variability in the shape and amplitude of the BOLDHRF correlates with endogenous GABAergic inhibition. Hum. Brain Mapp. 33, 455-465. doi: 10.1002/hbm.21223

Northoff, G., Walter, M., Schulte, R. F., Beck, J., Dydak, U., Henning, A., et al. (2007). GABA concentrations in the human anterior cingulate cortex predict negative BOLD responses in fMRI. Nat. Neurosci. 10, 1515-1517. doi: 10.1038/nn2001

Pan, W. J., Billings, J. C., Grooms, J. K., Shakil, S., and Keilholz, S. D. (2015). Considerations for resting state functional MRI and functional connectivity studies in rodents. Front. Neurosci. 9:269. doi: 10.3389/fnins.2015.00269

Paxinos, G., and Watson, C., (1998).The Rat Brain in Stereotaxic. Coordinates. San Diego, CA: Academic Press.

Poulet, J. F., Fernandez, L. M., Crochet, S., and Petersen, C. C. (2012). Thalamic control of cortical states. Nat. Neurosci. 15, 370-372. doi: 10.1038/nn.3035

Qiao, M., Rushforth, D., Wang, R., Shaw, R. A., Tomanek, B., Dunn, J. F., et al. (2007). Blood-oxygen-level-dependent magnetic resonance signal and cerebral oxygenation responses to brain activation are enhanced by concurrent transient hypertension in rats. J. Cereb. Blood Flow Metab. 27, 1280-1289. doi: $10.1038 /$ sj.jcbfm.9600436

Schaller, B., Xin, L., O’Brien, K., Magill, A. W., and Gruetter, R. (2014). Are glutamate and lactate increases ubiquitous to physiological activation? A (1)H functional MR spectroscopy study during motor activation in human brain at 7Tesla. Neuroimage 93(Pt. 1), 138-145. doi: 10.1016/j.neuroimage.2014.02.016

Seuwen, A., Schroeter, A., Grandjean, J., and Rudin, M. (2015). Metabolic changes assessed by MRS accurately reflect brain function during drug-induced epilepsy in mice in contrast to fMRI-based hemodynamic readouts. Neuroimage 120, 55-63. doi: 10.1016/j.neuroimage.2015.07.004

Sonnay, S., Just, N., Duarte, J. M., and Gruetter, R. (2015). Imaging of prolonged BOLD response in the somatosensory cortex of the rat. NMR Biomed. 28, 414-421. doi: 10.1002/nbm.3263

Stagg, C. J., Bestmann, S., Constantinescu, A. O., Moreno, L. M., Allman, C., Mekle, R., et al. (2011) Relationship between physiological measures of excitability and levels of glutamate and GABA in the human motor cortex. J. Physiol. 589(Pt. 23), 5845-5855. doi: 10.1113/jphysiol.2011.216978

Strupp, J. P. (1996). Stimulate a GUI based fMRI analysis software package. Neuroimage 3, S607. doi: 10.1016/S1053-8119(96)80609-4

Tiwari, V., Ambadipudi, S., and Patel, A. B. (2013) Glutamatergic and GABAergic TCA cycle and neurotransmitter cycling fluxes in different regions of mouse brain. J. Cereb. Blood Flow Metab. 33, 1523-1531. doi: 10.1038/jcbfm. 2013.114

Williams, K. A., Magnuson, M., Majeed, W., LaConte, S. M., Peltier, S. J., Hu, X., et al. (2010). Com-parison of alpha-chloralose, medetomidine and isoflurane anesthesia for functio-nal connectivity mapping in the rat. Magn. Reson. Imaging 28, 995-1003. doi: 10.1016/j.mri.2010.03.007

Xu, S., Yang, J., Li, C. Q., Zhu, W., and Shen, J. (2005). Metabolic alterations in focally activated primary somatosensory cortex of a-chloralose anesthetized rats measured by $1 \mathrm{H}$ MRS at 11.7 Tesla. NeuroImage 28, 401-409. doi: 10.1016/j.neuroimage.2005.06.016

Zhao, F., Zhao, T., Zhou, L., Wu, Q., and Hu, X. (2008). BOLD study of stimulation-induced neural activity and resting-state connectivity in medetomidine-sedated rat. Neuroimage39, 248-260. doi: 10.1016/ j.neuroimage.2007.07.063

Conflict of Interest Statement: The authors declare that the research was conducted in the absence of any commercial or financial relationships that could be construed as a potential conflict of interest.

Copyright (c) 2017 Just and Sonnay. This is an open-access article distributed under the terms of the Creative Commons Attribution License (CC BY). The use, distribution or reproduction in other forums is permitted, provided the original author(s) or licensor are credited and that the original publication in this journal is cited, in accordance with accepted academic practice. No use, distribution or reproduction is permitted which does not comply with these terms. 